\title{
Improving outpatient clinic attendance during the COVID-19 pandemic
}

\author{
Author: Kardo Ala-Aldeen ${ }^{\mathrm{A}}$
}

\section{Introduction}

Outpatient clinics have been severely restricted during COVID-19; the specialties face a tremendous challenge in service provision going forward. Attendance has been low in many centres despite implementation of COVID-19-related safety precautions. ${ }^{1}$ This study aimed to look at ways to improve clinic attendance during COVID-19.

\section{Methods}

Patients invited to the ophthalmology outpatient clinic at Luton and Dunstable Hospital between 22 June and 17 July 2020 were asked to give consent to complete a single telephone questionnaire. All patients that failed to attend their appointment were approached to take part. We also recruited a sample of 100 patients that attended clinic for comparison.

\section{Results}

546 patients were invited to clinic. Of these, 446 (71.7\%) attended their appointment and $100(18.3 \%)$ did not. 97 non-attenders were eligible for enrolment, of which $78(80.4 \%)$ consented to the study. $32 / 78(41 \%)$ did not attend for COVID-19-related reasons; of these $27 / 32(84.4 \%)$ were not aware of the COVID-19-related safety precautions in clinic. $23 / 78(29.5 \%)$ of non-attenders weren't aware of their appointment. $38 \%$ of attenders weren't aware of the safety precautions prior to their appointment. Following their visit, $95 \%$ of attenders felt that the safety precautions were excellent or satisfactory, and $99 \%$ would attend clinic again.

\section{Conclusion}

Our results show the need for enhanced communication to improve patients' awareness of COVID-19-related safety precautions and that clinics are still running. This is key to improving attendance and preventing NHS services becoming unsustainable during this pandemic.

\section{Conflicts of interest}

None declared.

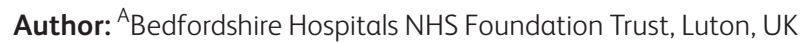

\section{Reference}

1 Royal College of Ophthalmologists. Guidance on restarting medial retina services. RCOpth, 2020. www.rcophth.ac.uk/wp-content/ uploads/2020/07/Guidance-on-restarting-Medial-Retina-Services. pdf [Accessed 08 September 2020]. 